\title{
Mit Nadeln gegen Kopfschmerzen
}

Bei Kopfschmerzen setzen Patienten auf Selbsthilfe und die Ganzheitsmedizin. Vor allem die Akupunktur, deren Auswirkung auf die Neurophysiologie nachweisbar ist, hat sich bewährt.

Neben Schmerzen im Bewegungssystem gehören Kopfschmerzen und Migräne zu den häufigsten Problemen, unter denen Patienten leiden. Die Prävalenz von chronischer Migräne wird etwa auf $17 \%$ bei Frauen und auf 6\% bei Männern geschätzt [1]. Bei normalen Kopfschmerzen liegt die Prävalenz zwischen 60 und $70 \%$; etwa 20\% der Betroffenen suchen deshalb einen Arzt auf. Mehr als die Hälfte der Patienten behandeln ihre Kopfschmerzen selber, und ein Drittel sucht in der Komplementärmedizin Hilfe.

Bei den ganzheitlichen Verfahren spielen vor allem die Phytotherapeutika eine wichtige Rolle. Bewährt hat sich zur Behandlung von Kopfschmerzen mithilfe der Hausapotheke das Pfefferminzöl, das auf die Stirn aufgetragen in seiner Wirkung der von Paracetamol entsprechen soll. Hagebuttenpulver oder Weidenrindenextrakte haben sich ebenfalls gut bewährt. Seit langem kommen Präparate aus dem Mutterkraut (Tanacetum parthenium) zum Einsatz. Der Wirkstoff ist Parthenolid, das die Seretonininhibition bewirkt. Grosse Hoffnungen hat man in die Wirkung von Tanacetum zur Prophylaxe der Migräne gesetzt. Allerdings kommt eine Cochrane-Studie [2] zu dem Schluss, dass hierfür keine ausreichenden Belege vorhanden sind.

Sehr gute Erfolge konnten in der Vergangenheit mit der Akupunktur bei der Behandlung von Kopfschmerzen und der Migräne erzielt werden. In einem Cochrane-Review [3] wurde die Wirkung der Akupunktur bei der Behandlung von Spannungskopfschmerzen untersucht. Dabei wurden 11 Studien mitinsgesamt etwa 2300 Patienten analysiert. Bei 47\% der Patienten, die mit Akupunktur behandelt wurden, verringerte sich die Zahl der Kopfschmerztage um über die Hälfte. Bei Patienten, die nur eine herkömmliche Grundversorgung erhalten hatten, waren es lediglich $16 \%$. In anderen Studien, die mit Kontrollgruppen gearbeitet haben, die eine Placebo-Akupunktur bekommen hatten, zeigte sich, dass in der Placebo-Gruppe 41\% der Patienten ihre Kopfschmerztage um 50\% reduzieren konnten - bei der Akupunkturgruppe waren es über 51\%. Die Autoren des Cochrane-Berichtes kommen zu dem Schluss, dass sich die Akupunktur wahrscheinlich für die Behandlung dieses Typs von Kopfschmerzen gut eignet.

In den letzten 2 Jahrzehnten hat sich die medizinische Forschung intensiv mit der Auswirkung der Akupunktur auf die Neurophysiologie des Menschen und das Schmerzsystem beschäftigt. Dabei standen insbesondere Prozesse im Mittelpunkt des Interesses, die mit der analgetischen Wirkung der Akupunktur in Verbindung stehen könnten.

Es zeigt sich, dass die Akupunktur für eine Aktivierung der A-Omegaund C-afferenten Fasern sorgt. Die afferenten Signale werden über das Rückenmark zum Mesencephalon und zum Hypothalamus übertragen. Dabei wird eine Reihe von Mediatoren ausgeschüttet, die eine analgetische Wirkung besitzen. Dazu gehören Opioide wie Dynorphin oder Enkephalin. Im Hypothalamus wird das adrenokortikotrope Hormon ausgeschüttet, das den adrenalen Kortex stimuliert und für die Ausschüttung von Kortikoglykosiden sorgt. Neurotransmitter, die einen in- hibitorischen Einfluss auf die post- und präsynaptische Nervweiterleitung haben und damit in das zentrale Schmerzweiterleitungssystem eingreifen, werden bei der Akupunktur vermehrt freigesetzt. Dazu gehören die Norepinephrine Serotonin und Dopamin.

Bei der Elektroakupunktur können je nach Frequenz unterschiedliche neurophysiologische Effekte verursacht werden. Bei der niederfrequenten Elektroakupunktur werden vor allem Enkephaline und BetaEndorphine ausgeschüttet, bei der hochfrequenten Elektroakupunktur vor allem Dynorphin.

Ein sehr starker Hinweis, dass für die Wirkung der Akupunktur tatsächlich neurophysiologische Effekte und biochemische Vorgänge verantwortlich sind, zeigen die Versuche von Han [4]. Dabei wurde Tieren Cerobrospinalflüssigkeit entnommen und auf andere Tiere übertragen. Damit liess sich ein analgetischer Effekt mit übertragen. Auf eine starke biochemische Basis der Akupunktur deuten auch andere Versuche hin, bei denen Opioid-Antagonisten eingesetzt wurden. Durch die Gabe von Naloxon konnte die analgetische Wirkung der Akupunktur wieder rückgängig gemacht werden.(rfi)

\section{Literatur}

1 Dhanani NM, Caruso TJ, Carinci AJ: Complementary and alternative medicine for pain: an evidence-based review. Curr Pain Headache Rep 2011;15:39-44.

2 Pittler MH, Ernst E: Feverfew for preventing migraine. Cochrane Database Syst Rev 2004;1: CD002286.

3 Cheng RS, Pomeranz BH: Electroacupuncture analgesia is mediated by stereospecific opiate receptors and is reversed by antagonists of type I receptors. Life Sci 1980;26:631-638.

4 Han JS: Physiology of acupuncture: review of thirty years of research. J Altern Complement Med 1997;3(suppl 1):101-108.

\section{KARGER}

Fax +49 7614520714 